\title{
Autonomous Traffic Signal Control using Decision Tree
}

\author{
Rithesh R. N., Vignesh R, Anala M. R.
}

R V College of Engineering, Bangalore, Indonesia

\begin{tabular}{l} 
Article Info \\
\hline Article history: \\
Received Oct 17, 2017 \\
Revised Dec 26, 2017 \\
Accepted Jan 3, 2018 \\
\hline
\end{tabular}

Keyword:

Background subtraction

Decision tree

Object detection

Traffic congestion

Vehicle count

Corresponding Author:

Anala M. R.,

Departement of Computer Science and Engineering,

R V College of Engineering,

R.V. Vidyaniketan Post, Mysuru Road, Bengaluru - 560059, India.

Email : analamr@rvce.edu.in

\begin{abstract}
The objective of this paper is to introduce an effective and efficient way of traffic signal light control to optimize the traffic signal duration across each lanes and thereby, to minimize or completely eliminate traffic congestion. This paper introduces a new approach to resolve the traffic congestion problem at junctions by making use of decision trees. The vehicle count in the real time traffic video is determined by Image Processing technique. This information is fed to the decision tree based on which the decision is made regarding the status of traffic signal lights of each lane at the junction at any given instant of time.
\end{abstract}

Copyright $\odot 2018$ Institute of Advanced Engineering and Science. All rights reserved.

\section{INTRODUCTION}

The primary intension of the traffic signal lights is to facilitate the transportation or movement of the vehicles in junctions without creating any chaos or adversely affecting the normal day to day life of people. Efficient working of the same is an essential part for smooth transportation, especially in densely populated metropolitan cities. But in today's age of globalization the number of vehicles stepping onto the road has elevated by significant amount leading to traffic congestion problem. Traffic congestion has emerged as one of the most serious problems faced by most of the countries today. It has adverse effect on the physical and mental health of the people, the environment, and is one of the main reasons for the cause of accidents. All of these problems can be minimized and in some cases completely eliminated by incorporating the right methodology which controls and coordinates flow of vehicles on the road in an efficient manner, and thereby makes optimum utilization of time and available space for vehicle transportation. This paper introduces an efficient way for traffic signal light control using decision trees. The decision tree modeled here is mainly based on the number of vehicles on each lane at the junction. The count of the number of vehicles across each lane at the junction is obtained by using Image processing techniques. The decision regarding the status (i.e. color) of the signal lights across each lanes of the junctions is made based on the number of vehicles on each lanes and, accordingly the status of the signal lights are changed in real time to achieve maximum throughput in terms of number of vehicles clearing/crossing the junction, with minimum chances of traffic jam.

\section{LITERATURE REVIEW} control.

Intelligent transportation systems (ITS) mainly aim at providing better quality of traffic congestion 
The world has seen huge advancements in ITS some of them are follows:

A methodology in which the system detects and controls the traffic congestion in real time based on the extent to which congestion has occurred by employing active RFID (gateway, router and tags) and GSM technology is discussed in [1]. This method requires each vehicles to have RFID tag installed in them and based on the relative speeds of the vehicles the traffic signal is controlled by the coordinators. This technique requires a huge initial investment for system setup and, difficult to implement in cities with high congestion problem because the traffic congestion at given signal not only affects the signal lights of this junction but also influences the signal lights of previous junctions. Therefore due to chain reaction, in the worst case a series of signals can experience a severe congestion problem.

The technique in which traffic signal control is done using image processing techniques is presented in [2]-[4]. The similarity between a reference image of a road and the real image of same road becomes the criteria for traffic signal light control. Higher the similarity lower is the number of vehicles and viz. Preference is given to road which has least similarity value. This technique takes into account only the similarity value therefore irrespective of number of vehicles across each lanes GREEN signal is given to only those lanes with least similarity value. There might occur a situation in which one lane has fewer number of Large Motor Vehicles(LMV) in which the similarity value is small and another lane might have greater number of small sized vehicles covering less area across the road compared to the road with LMV thus similarity value would be comparatively high. In this case former lane is given GREEN signal and later case is given RED signal thus failing to avoid congestion at the densely populated roads.

A methodology in which the vehicle count is determined using Image processing technique and a learning model is designed which predicts the duration of traffic signal for the next iteration based on the available data of current iteration, is presented in [5]. Also, [6] and [7] proposes alternative techniques to overcome traffic congestion and traffic accidents.

\section{RESEARCH METHOD}

The proposed methodology encompasses two main phases:

a. Object detection and vehicle counting

b. Decision making using Decision tree.

Each of these phases is explained in detail in the following section.

\subsection{Object detection and vehicle counting}

A camera capable of capturing all the lanes at a given junction has to be setup. The position and orientation of this camera should be consistent in giving the proper view of all the lanes. A balanced pictorial view of all the lanes obtained from the camera helps in achieving better results for decision making process. This real time video from this camera is given as input to the microcontroller/microprocessor. The Image processing technique for object detection and counting and, the algorithm for decision making is programmed in the microprocessor. The image processing technique used here follows extraction of frames from the video, Background subtraction, threshold setup, creating contours, blob detection, and vehicle counting which is more accurate than the object detection technique presented in [8] and [9]. The captured video is broken down into sequence of frames, the frames are extracted at a rate of 25 frames/sec. These sequences of frames are processed to detect multiple moving objects (vehicles). For the purpose of explanation, the algorithm is applied for a two lane traffic junction. At the beginning of this algorithm the vehicle count across all the lanes is initialized to zero. A virtual line is drawn across each lanes at the junction to keep track on the number of vehicles waiting to cross the junction. The virtual lines are considered as the line of reference for the movement of the vehicles. Figure 1 shows original image extracted from the video and the image with virtual lines draws across each of the lanes of the junction.

The extracted frames from the video are converted into binary black and white image. It is evident from [10] that Canny Edge detection is the most efficient technique for object edge extraction, hence the same technique is used here. Contours are drawn for the binary image and convex hulls are constructed with the help of these contours and, finally blobs are formed. Each of valid blobs are added to the list list_blobs. For each and every blob in the list list_blobs, if the blob has crossed the virtual line then the vehicle counter associated with the corresponding lane is incremented. The blobs may be categorized as 2-wheeler or 4wheeler based on the size and aspect ratio. And also two separate counters may be assigned to each lane at the junction, one to account for number of two wheeler vehicles waiting to cross the junction and the other for four wheeler. Crossing the line merely refers to the fact that the midpoint of the blob under reference is on one side of the line in the previous frame and in the current frame the midpoint is on or has crossed the virtual line. In Figure 2 for each of the lanes two counters are maintained respectively. One for the 2 wheeler vehicle and the other for the 4 wheeler vehicle as shown in the top left corner for lane 1(Left lane in Figure 2) 
and bottom right corner for lane 2(Bottom lane in Figure 2), in yellow color. In the pair of numbers for each lane, the upper number indicates the number of two wheeler vehicles and lower number indicates the number of four wheeler vehicles which have crossed the virtual line. The respective counters are refreshed every time the decision is made, in order to have a valid count for the next turn of decision making.

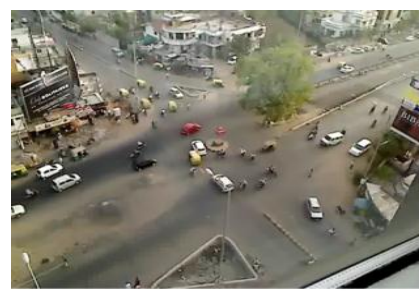

Figure 1. Extracted frame from video

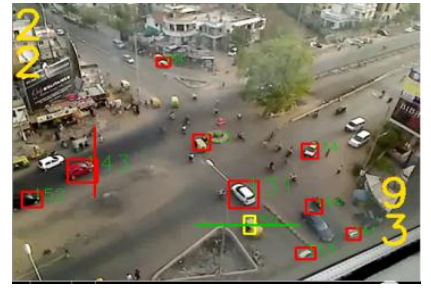

Figure 2. Output_frame

\subsection{Decision making using decision tree}

Basically decision tree is formed by posing series of questions about the characteristics or attributes of the input record, one after the other. The process starts from the root node, the test condition is applied to it in an internal node, and based on the outcome, appropriate branch is followed. This will take the input either to the next internal node where again a test condition is to be answered, or the leaf node that has a class label to which the input is classified to. Figure 3 shows the decision tree.

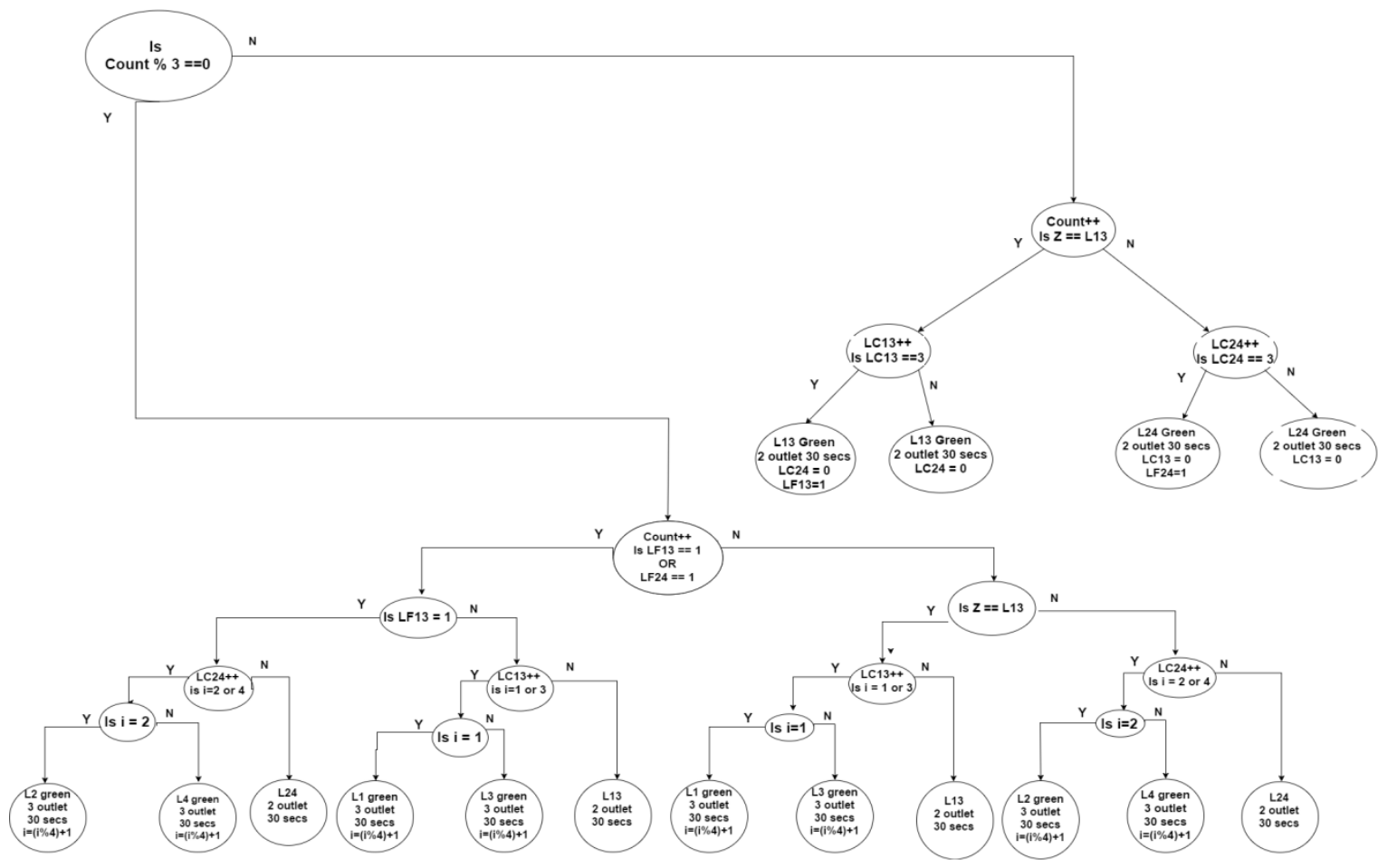

Figure 3. Decision tree

In Figure 3, ' $i$ ' stands for preferred lanes turn e.g. if $i=1$ then preferred lane is 1 , i.e. lane 1 will be given prime importance over other lanes for next three time slots ( 1 time slot $=30$ secs). 'count' stands for count on iteration, 'count' can be 0,1 or 2 . Every time the root node checks if the Lane $i$ 's three iteration (i.e. three time slots) is completed, If completed then next lane is chosen as the preferred lane and the status of the lane flag is checked. Else, ' $i$ ' is incremented and respective lane count (lane counts give the number of consecutive times the respective lane was given green signal) is incremented. If the three iterations are over 
and in all the 3 consecutive iterations if same set of lanes are given green signal then respective lane flags are set. At level 2 of left sub-tree, status flag and $\mathrm{Z}$ is checked based condition at level 1 . After a couple of similar conditions the decision is taken at the leaves.

Each leaf node of the tree makes the decision on which of the following classes does the given input (L13 \& L24) belongs to:

Class L1: Lane 1 is given green for 30 seconds and Lane 2,3 and 4 are red.

Class L2: Lane 2 is given green for 30 seconds and Lane 3,4 and 1 are red.

Class L3: Lane 3 is given green for 30 seconds and Lane 4,1 and 2 are red.

Class L4: Lane 4 is given green for 30 seconds and Lane 1,2 and 3 are red.

Class L13: Lane 1 and Lane 3 are given green for 30 seconds and Lane 2 and Lane 4 are given red.

Class L24: Lane 2 and Lane 4 are given green for 30 seconds and Lane 1 and Lane 3 are given red.

The discriminant function $\mathrm{g}(\mathrm{x}, \mathrm{y})$ is defined as follows:

$$
\mathrm{Z}=\mathrm{g}(\mathrm{x}, \mathrm{y})=((\mathrm{y} / \mathrm{x})-1)
$$

Here, $\mathrm{y}$ is total number of vehicles across lane 2 and 4 and,x is total number of vehicles across lane 1 and 3.

\section{Decision rule:}

If $(Z>0$ AND $Z<10)$ OR $(Z<-0.1)$

Then decide GREEN signal for lane 2 and 4

(i.e. decide L24)

If $(\mathrm{Z}<0$ AND $\mathrm{Z}>-0.1)$ OR $(\mathrm{Z}>10)$

Then decide GREEN signal for lane 1 and 3

(i.e. decide L13)

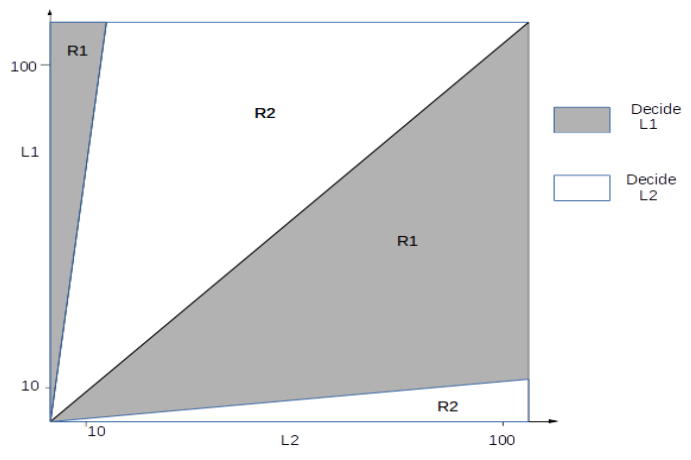

Figure 4. discriminant region

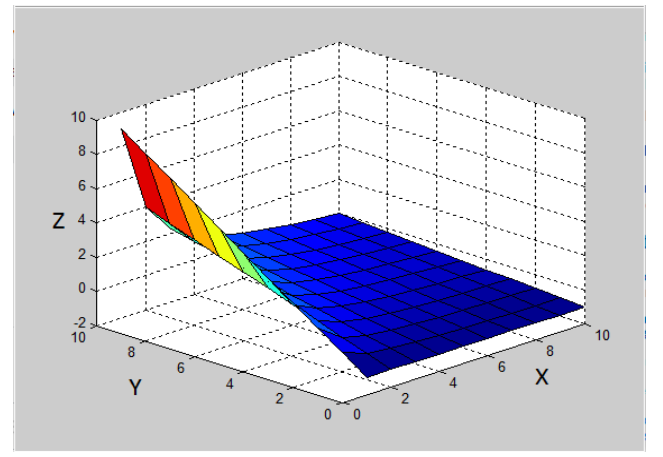

Figure $5 . \mathrm{Z}=\mathrm{g}(\mathrm{x}, \mathrm{y})$

Graph shown in Figure 4 is a 2D graph depicting the regions R1 (gray) and R2 (white), Count of number of vehicles across lane 1 and 3 is shown across $x$-axis and number of vehicles across lane 2 and 4 is shown across y-axis, Figure 4 shows the picturization as to how the different combination of vehicles count are bifurcated as belonging to region 1 and region 2 respectively. Graph shown in Figure 5 presents the discriminant function $Z$, with $x$ and $y$ axis depicts the number of vehicles across L13 (=L1+L3) and L24 $(=\mathrm{L} 2+\mathrm{L} 4)$ respectively, and $\mathrm{Z}$ is along $\mathrm{Z}$ axis. The decision rule is applied over the $\mathrm{Z}$ value obtained for the given set of inputs, and finally decision is made. The algorithm for Decision tree in autonomous traffic signal control is given below.

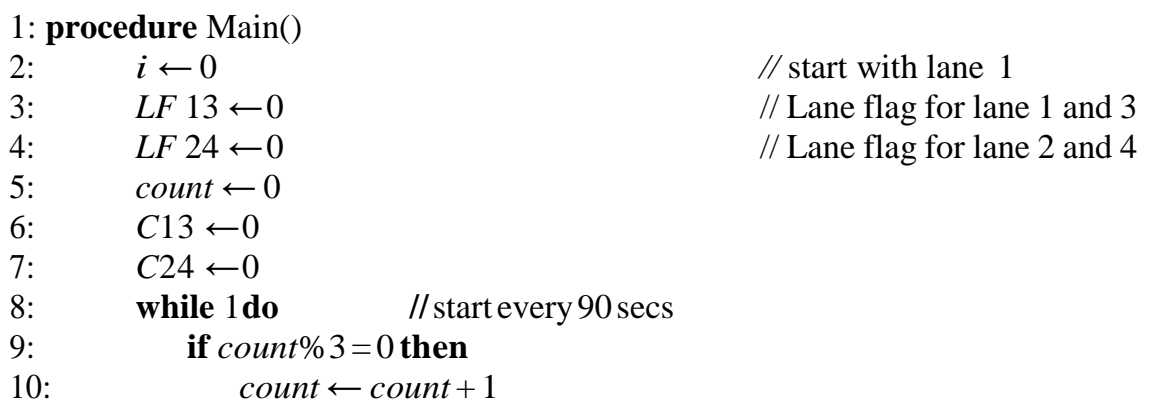




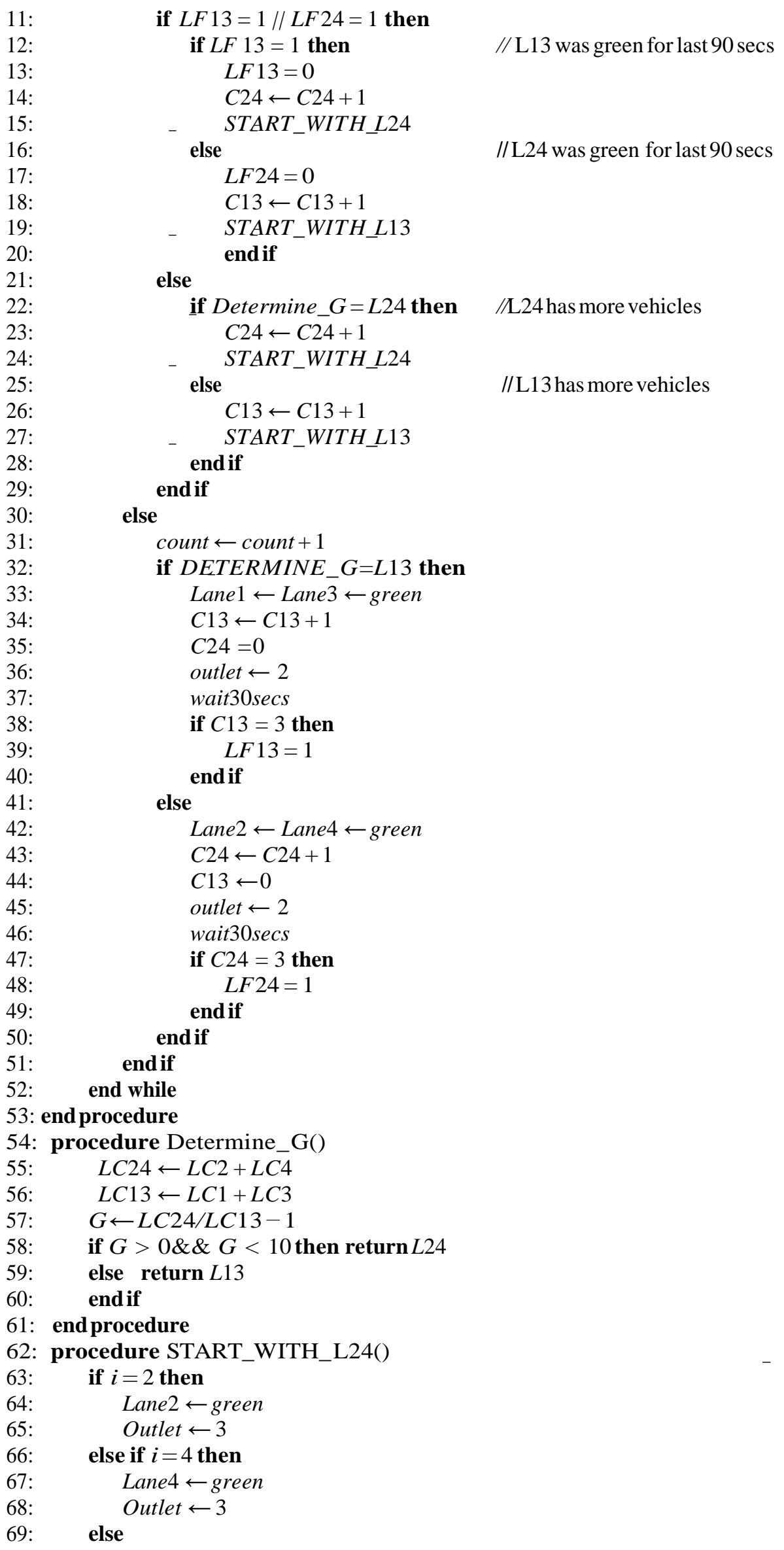




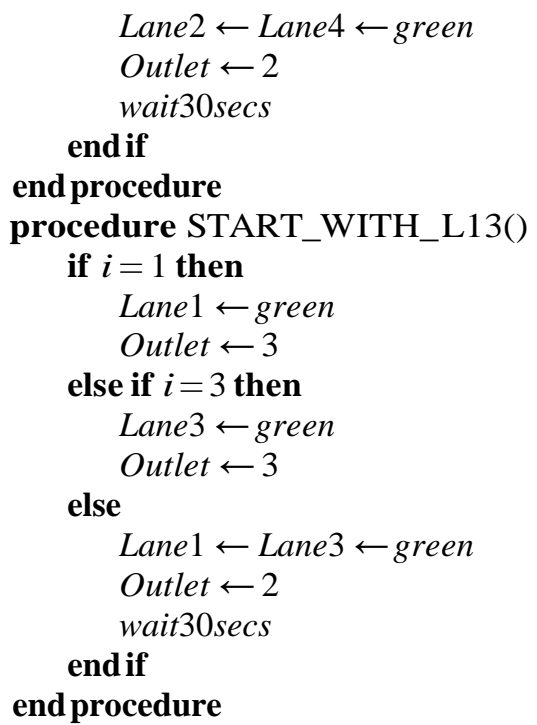

In this context, the input to the decision tree are the features of 4 lanes L1, L2, L3 and L4. These features include the vehicle count of each lane and a flag associated with the pair of lanes i.e. LF13 and LF24.The flag value 1 for a pair of lanes specifies that they were given green signal for 3 consecutive time (30 sec each). The leaf nodes specifies which lanes should be given with the green signal for the next 30 secs and also marks the flags of the lanes if they were given green signals for 3 consecutive times which is used as the input to the next iteration.

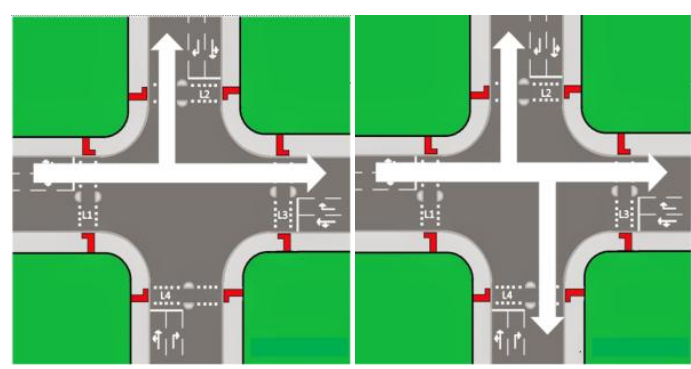

Figure 6.3 outlet format and 2 outlet format

3 outlet format: In this format, a selected lane (say L1) allows its vehicles to move in 3 directions i.e straight (along lane3), left (along lane2) and right (along lane4).

2 outlet format: In this format, a selected lane (say L1) allows its vehicles to move in 2 directions i.e straight (along lane3) and left (along lane2). Figure 6 represents both these formats.

The tree is executed once in every time slot $(30 \mathrm{secs})$. First we check if 90 seconds (i.e three $30 \mathrm{sec}$ time slots) is completed using the condition count $\% 3=0$. if it has then we check if any pair of lanes (L13 or L24) were given green signal for 90 secs i.e. for 3 continuous slots. If that's true (say L13 was green for 90 secs), then irrespective of the result of the $\mathrm{Z}$ function, the other two lanes (i.e. L24) are given the preference so as to avoid starvation. If none of the flags were set, the function $\mathrm{Z}$ is called to decide which of the pairs is to be given preference for the first 30 secs (Assume $\mathrm{Z}$ decides L24). So for the first 30 secs, one of these two lanes (L2 or L4) has to be given green signal with 3 outlet format, based on 'i' value. The lane which has its turn in this iteration is selected here. ie, if L2 is preferred lane in this iteration then L2 is selected or if L4 has its turn then L4 is selected. If neither of them had its turn, i.e L1 or L3 had its turn in the corresponding iteration, but based on Z L24 got green then both of them are given green with 2 outlet format. In the second and third time slots only 2 outlet format is followed based on pair of lanes chosen by Z. If count $\% 3$ is not 0 , i.e. 90 secs is not completed, then the pair of lanes is selected purely based on the result of function Z. Here is where we check if a pair is being given green signal for 3 consecutive times or 90 consecutive seconds and thereby set the corresponding flags to avoid starvation of the other pair of lanes. For a 4 lane structure of the decision tree, the following methodology is followed: 
For every iteration (i.e every $30 \mathrm{secs}) \mathrm{L} 13(=\mathrm{L} 1+\mathrm{L} 3)$ and L24 ( = L2+L4) is computed. Every lane (In this case 4 lanes) meeting at the junction is given equal priority in Round Robin fashion, i.e every lane becomes preferred lane (gets 3 outlet format for 1st 30secs) within a maximum of 11 time slots and irrespective of the magnitude of congestion at the traffic junction every lane get green signal (2 outlet format) within a maximum of 6 time slots. After every 3 iterations, a lane ' $\mathrm{i}$ ' becomes preferred lane based on the following rule

$$
\mathrm{i}=(\mathrm{i} \% 4)+1
$$

\section{RESULTS AND ANALYSIS}

The algorithm is implemented in $\mathrm{C}++$ in visual studio along with openCV open source software. The vehicle detection and counting algorithm was executed for 2 simulation videos and 3 real time videos, each for a span of 60 seconds and the following results were obtained. Actual count column in the Table. 1 signifies the actual number of vehicles in the video this count was obtained by manually counting the vehicles. Count obtained column gives the number of moving vehicles identified by the algorithm, the corresponding deviation and accuracy is mentioned in the Table 1. The implementation of this technique consists of two different programs running simultaneously, one program constitutes the image processing technique for moving vehicle detection and counting and, the other program is for making decision on status of traffic signal light based on decision tree. The output of the former program is written in text file (input) and the later program which is running simultaneously reads this text file (input) and makes decision on traffic signal.

The output of the later program can be written to another text file or can be fed to control switches of traffic lights. The model designed here is for the autonomous traffic control, thus the number of vehicles across each of the lanes is determined and instantaneously written to the text file .This file is read continuously by decision tree and correspondingly the status of the traffic lights is varied based on the real time situation of the junction.

Table 1. Result Analysis

\begin{tabular}{cccccc}
\hline Sl. no & Actual count & Count obtained & Deviation & Accuracy & Nature of video \\
\hline 1 & 52 & 52 & $0 \%$ & $100 \%$ & Real \\
2 & 118 & 92 & $22 \%$ & $78 \%$ & Real \\
3 & 72 & 72 & $0 \%$ & $100 \%$ & Simulation \\
4 & 51 & 60 & $17.50 \%$ & $82.50 \%$ & Simulation \\
5 & 109 & 106 & $0.02 \%$ & $99.98 \%$ & Real \\
\hline
\end{tabular}

The accuracy of the result is mainly dominated by quality of the video and the camera orientation.

The variation or difference between the actual vehicle count and the vehicle count obtained in traffic video is attributed to the following factors:

a. Noise in the video- because of which most of the information is inconsistent and object detection becomes more difficult. Applying denoising algorithm to each frame in the video tends to be time consuming and results in loss of useful information.

b. Distance factor- when the group of vehicles are very close, the algorithm considers it as one single object and thus the counting cannot be made $100 \%$ accurate.

c. Quality of the video

d. Placement/Position of the camera with respect to the cross road/junction.

Table 2. Decision Tree Results

\begin{tabular}{ccccc}
\hline $\begin{array}{c}\text { Time } \\
\text { stamp(TS) }\end{array}$ & $\begin{array}{c}\text { Lane1 } \\
\text { (vehicle count) }\end{array}$ & $\begin{array}{c}\text { Lane2 } \\
\text { (vehicle count) }\end{array}$ & Decision & Output \\
\hline 30 & 100 & 50 & L1 & L1 green for 30 secs \\
60 & 120 & 70 & L1 & L1 green for 30 secs \\
90 & 110 & 80 & L1 & L1 green for 30 secs \\
120 & 135 & 85 & L2 & L2 green for 30 secs \\
150 & 140 & 10 & L1 & L1 green for 30 secs \\
180 & 30 & 20 & L1 & L1 green for 10 secs \\
210 & 5 & 15 & L2 & L2 green for 30 secs \\
240 & 10 & 105 & L1 & L1 green for 30 secs \\
\hline
\end{tabular}


Table 2 describes the result of decision tree for a junction with 2 lanes. Lane 1 is given green signal thrice each with time slot of 30 seconds. In the next turn even if L1 has more number of vehicle than L2, still L2 is set to green thereby avoiding starvation. At time stamp 240 though L2 has more vehicles than L1 still L1 is given green signal in order to avoid small number of vehicles waiting for a relatively longer duration of time.

\section{CONCLUSION}

The model designed for autonomous traffic signal control is coherent with its purpose. The methodology implemented here is completely based on number of vehicles across each lanes and, since traffic congestion directly depends on number of vehicles waiting to cross junction across each lane. This technique minimizes the traffic congestion, and in some cases it is completely eliminated. The statistics on the vehicle count can also be used for the infrastructural development such as flyovers, metro etc. The decision tree designed is a generic model and can be extended to any number of lanes by making appropriate changes in different levels of the decision tree. The worst case scenario is when a set of vehicles of a lane has to wait for 5 continuous slots or 150 secs. This seems highly impractical in reality. People can't know when exactly they will be allowed to move as the decision is taken at run time at the end of 30 secs or a slot.

\section{REFERENCES}

[1] Siuli Roy, Somprakash Bandyopadhyay, Munmun Das, Suvadip Batabyal, Sankhadeep Pal, "Real Time Traffic Congestion Detection and Management using Active RFID and GSM Technology", In proc. of the 10th International Conference on Intelligent Transport Systems Telecommunication (ITST'10).

[2] Nilay Mokashi, "Intelligent Traffic Signal Control using Image Processing", International Journal of Advanced Research in Computer Science and Management Studies, vol. 3, no. 10, 2015, ISSN: 2321-7782.

[3] Susmita A.Meshram, A.V. Malviya, "Traffic Surveillance by Counting and Classification of vehiclesfrom video using Image processing", International Journal of Advanced Research in Computer Science and Management Studies, vol. 1, no. 6, 2013, ISSN: 2321-7782

[4] Ms. Pallavi choudekar, Ms.b Sayanti Banerjee, Prof M.K. Muju, "Real time traffic light control using image processing", Indian Journal of Computer Science and Engineering, vol. 2, no. 1, ISSN: 0976-5166, pp. 6-10.

[5] Archit Peshave, Shantanu RajeNimbalkar, Ajinkya Puar, Vikas Gardare, Abhijeet Dodake, Jitendra Waydande, "A Review on Autonomous Traffic Lights Control System", International Journal of Innovative Research in Computer and Communication Engineering, vol. 3, no. 10, October 2015, pp. 10034-10037.

[6] Sutikno, Helmie Arif Wibawa, Prima Yusuf Budiarto, "Classification of Road damage from digital Image using Backpropagation Neural Network", IAES International Journal of Artificial Intelligence, vol. 6, no. 4, December 2017, ISSN: 2252-8938.

[7] Kusuma Kumari, Sampada Sethi, Ramakanth Kumar, Nishant Kumar, Atulit Shankar, "Driver Drowsiness Detection System using Sensors", IAES International Journal of Informatics and Communication Technology, vol. 6 , no. 3 .

[8] Varun Sharma, "Object Counting using MATLAB”, International Journal of Scientific \& Engineering Research, vol. 5, no. 3, March 2014. ISSN 2229-5518.

[9] Ganesh Raghtate, Abhilasha K Tiwari, "Moving Object Counting in Video Signal”, International Journal of Engineering Research and General Science, vol. 2, no. 3, 2014, ISSN: 2091-2730.

[10] Brinda R.B, Namratha Venkatesh Murthy, B.M. Ramya, Dr. Vijaya Prakash A M, "Edge detection Smart Traffic Control", International Journal of Innovative Research in Electrical, Electronics, Instrumentation and Control Engineering, vol. 3, no. 11, 2015, ISSN: 2321-2004. 